\title{
A Chronic Disease Management Model for Chronic Liver Failure
}

\author{
Alan J. Wigg, ${ }^{1,2}$ Mohamed A. Chinnaratha, ${ }^{1,2}$ Rachel Wundke, ${ }^{1}$ and Michael L. Volk ${ }^{3}$
}

\section{Editor's Note}

Clinical Perspectives in Hepatology aims to engage experts with opinions supporting differing perspectives on the management of a case. Typically, the case represents an area of debate or evolving practice in clinical hepatology. The case described by Drs. Wigg, Chinnaratha, and Registered Nurse Wundke provides an opportunity to discuss the opportunities and challenges associated with chronic disease management models for patients with endstage liver disease.

Chronic Disease Management (CDM) is a patientcentered approach to healthcare delivery that emphasizes proactive, longitudinal care between visits. Most healthcare systems are still based on acute illness disease models established at the beginning of the 20th century. In contrast, chronic illnesses now account for more than $75 \%$ of total healthcare expenditures. ${ }^{1}$ The episodic, symptomdriven nature of healthcare systems is poorly structured to manage this increasing burden of chronic conditions. Chronic liver failure (CLF) is one such condition, with a median time from diagnosis to death of 10 years, high morbidity, and frequent hospitalizations. ${ }^{2,3}$ In similar conditions such as heart failure, advances in CDM have substantially improved patient outcomes. These advances include relatively simple concepts such as patient education, medication reconciliation, and telephone management between visits. ${ }^{4}$ Recent pilot data suggest that similar interventions might also improve outcomes in cirrhosis. ${ }^{5,6}$

An Australian randomized controlled pilot trial $(\mathrm{RCT})$ did not demonstrate significant differences in

Abbreviations: CDM, chronic disease management; CLF, chronic liver failure; MELD, Model for Endstage Liver Disease.

From the ${ }^{1}$ Hepatology and Liver Transplantation Medicine Unit, Flinders Medical Centre, Adelaide, Australia; ${ }^{2}$ Flinders University of South Australia, Adelaide, Australia; ${ }^{3}$ Gastroenterology and Hepatology, University of Michigan Health System, Ann Arbor, MI.

Received March 7, 2014; accepted March 27, 2014.

Address reprint requests to: Dr. Alan J. Wigg, Hepatology and Liver Transplantation Medicine Unit, Flinders Medical Centre, Bedford Park, 5042, Australia.E-mail: alan.wigg@health.sa.gov.au; fax: + 61882043943.

Copyright $(2014$ by the American Association for the Study of Liver Diseases.

View this article online at wileyonlinelibrary.com.

DOI 10.1002/hep.27152

Potential conflict of interest: Dr. Volk received grants from Salix. the hospital admission rates, severity of liver disease, or quality of life. ${ }^{5}$ However, benefits in outpatient clinic attendance and quality of care were observed. A second nonrandomized Italian study of 100 patients found reduced hospitalization, mortality, and costs in the intervention group. ${ }^{6}$ The following case report provides a demonstration of CDM principles and how they can be applied to a CLF patient. The response to the case and discussion offers an approach to advance the field of CDM models for CLF.

\section{Case Presentation by Drs. Wigg, Chinnaratha, and Registered Nurse Wundke}

A 38-year-old female first presented to our hospital in 1998 with alcohol intoxication and benzodiazepine overdose. The following 10 years were characterized by multiple presentations to the emergency department, some requiring prolonged in-patient hospital stays. The main indications for admission were related to alcoholic hepatitis and polypharmacy overdose with suicidal intent. Her chronic alcohol abuse, chronic hepatitis $\mathrm{C}$ infection, and depression could not be curtailed by either general practitioners or hospital consultants due to chronic nonadherence with medications, counseling sessions, and out-patient visits.

In late 2008 she presented with recurrent falls and hematemesis. Assessment showed grade 3 encephalopathy, jaundice, and Model for Endstage Liver Disease (MELD) score of 38. Gastroscopy showed bleeding esophageal varices, which were banded. She was managed with nasogastric feeding and supportive measures.

Prior to discharge, she was enrolled into a CDM program comprised of coordinated case management by a nurse, supervised by a hospital-based hepatologist. Specific examples of interventions included: regular home visits, frequent telephone reviews, rapid access to care pathway by way of call to a nurse mobile phone, patient action plans for ascites and encephalopathy, and medication blister packs.

Hospital usage following enrolment is shown in Figs. 1 and 2. The initial year postenrolment showed ongoing high levels of emergency room presentations 


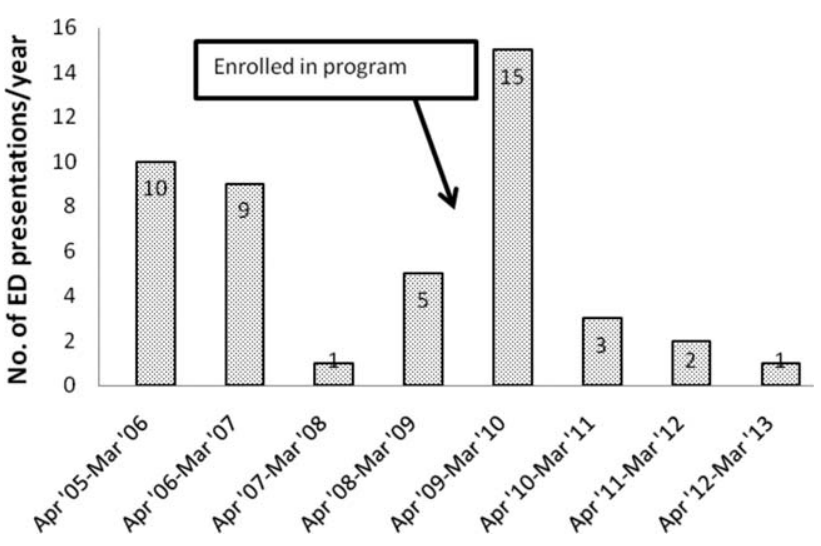

Fig. 1. Emergency department (ED) presentations pre- and postenrolment.

and inpatient hospital days. However, there were dramatic declines in these indices for the following 3 years. The patient's attendance at alcohol counseling sessions, psychiatry, and outpatient clinic visits improved, along with better medication compliance. Alcohol abstinence was achieved and has been maintained for 2 years and the current MELD score is 11 .

\section{Dr. Wigg and Colleagues' Perspective}

While it is intuitive to believe that models that have been successfully used for other chronic diseases will work in CLF, this should not be assumed. CLF may be a more challenging disease for CDM interventions, due to the heterogeneity of etiologies and clinical manifestations, the severity of the disease, and the frequent association with substance abuse. As suggested by this report, one of the key interventions required for success is case-managementcharacterized by frequent, accessible, and long-term contacts between the patient and coordinators to help overcome many of the structural barriers that prevent effective interactions with clinicians in both hospital and primary care. This arrangement can improve therapeutic relationships for the patient from which can flow greater engagement with multidisciplinary teams, improved self-management, and improved adherence with evidence-based therapies. The case also suggests the need for long-term commitment in order to achieve benefits, which may not be seen in early phases of the intervention.

Another possibility to consider is that increased early hospitalization may be a consequence of improved access to care and an indicator of quality care. ${ }^{7}$ Perhaps increased early hospitalization is required to help keep sick patients alive, and will translate downstream into relative mortality reductions. For this reason mortality is likely to be the key endpoint in CDM studies of cirrhosis.

Alternative approaches to care redesign have been studied in cirrhosis. The "Care Management Program" and "Day Hospital" model in which investigations are performed, reviewed, and care plans formulated, all during a 1-day admission, has shown promise in a nonrandomized single-center study. ${ }^{6}$ The availability of a day hospital facility for day admissions such as paracentesis, frequently required by cirrhosis patients, is also likely to improve the efficiency of hospital utilization.

Greater involvement of primary care physicians also has potential to improve patient care. Traditional hospital-centered models led by gastroenterologists usually fail to provide adequate education and support of primary care physicians, with whom patients spend the majority of their healthcare time. Models that provide greater collaboration and education of primary healthcare clinicians have shown benefit in the treatment of hepatitis C patients ${ }^{8}$ and should be incorporated into future CDM models in CLF. Practical examples of this could include videoconferencing between hospital and primary care teams during care plan formulation and discharge planning.

\section{Dr. Volk's Perspective}

Although CDM holds enormous promise, the case presented by Dr. Wigg and colleagues highlights the difficulties with implementing disease management in clinical practice. One obvious barrier to $\mathrm{CDM}$ is the payment structure of healthcare systems, which rarely cover proven treatments that are not pharmaceutical or procedural in nature. The personnel effort described in this case was funded through a clinical trial, and such resources are not currently available to most healthcare providers. The introduction of Accountable Care Organizations in the United States will hopefully

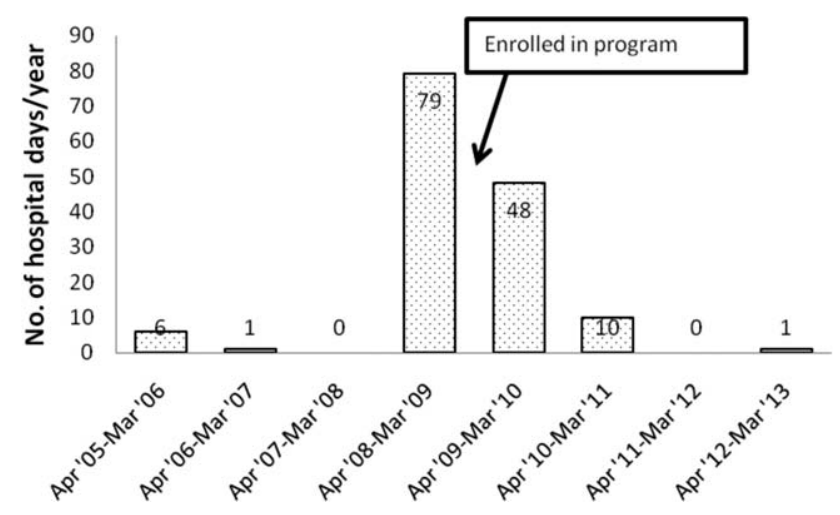

Fig. 2. Days spent in hospital pre- and postenrolment. 
provide health systems with financial incentives to pay for CDM interventions, through shared savings of any resultant decrease in healthcare expenditures. ${ }^{\text {? }}$

A second, more fundamental, barrier is translating results from clinical trials into practice. Unlike the prescription of a new pill, implementation of CDM interventions require setting up new protocols and training staff, and can be lost in translation from one clinical setting to another. I suspect the patient described in this case benefited from a highly skilled and experienced hepatology nurse, and such individuals do not grow on trees!

How, then, can the field of hepatology move forward with CDM? I believe we need to distinguish between clinical trials, implementation trials, and quality improvement. Traditional RCTs focus on testing specific interventions in a scientifically rigorous manner, but do not test how the intervention is formulated and delivered. Unlike a pill, CDM interventions cannot be mass-produced in a factory and shipped all over the world. Therefore, CDM trials should probably borrow from the field of Implementation Science, which focuses not only on effectiveness of the intervention but also on the effectiveness of the implementation process. ${ }^{10}$ For example, if the intervention includes telephone management by a nurse, then the intervention should include training protocols and scripts, and the study should specifically measure the effectiveness of training inexperienced nurses to administer the intervention. Finally, even interventions with proven effectiveness and proven implementation methods may need to be adjusted to fit different clinical settings. Here is where quality improvement comes in. Just as many hepatology programs track their rates of sustained virological response (SVR) to antiviral therapy for hepatitis $\mathrm{C}$ and adjust their protocols accordingly, programs implementing CDM protocols should monitor for metrics of effectiveness-such as improvements in patient knowledge or self-efficacy. ${ }^{11}$

\section{Dr. Wigg and Colleagues' Reply}

We thank Dr. Volk for raising the above practical questions. CDM model implementation will be associated with costs (coordinators, improved adherence, improved survival). It is therefore critical that future studies rigorously evaluate the incremental cost effectiveness of these models relative to usual care. Such analysis will enable us to understand whether there are cost savings or that increased costs are associated with acceptable thresholds of cost per quality adjusted life year. Establishing cost effectiveness in clinical trials is likely to be a helpful driver of change to current funding models of healthcare with movement towards financial incentives for management of complex chronic diseases, such as payment for video-conferencing and care planning.

Translating results from clinical trials of CDM into real-life practice will be a significant challenge for this form of complex treatment. The design of trials with generalizability to routine care will therefore be a priority. This will require multicenter trials and testing of the model in a variety of different clinical settings, including the common setting of community hospitals where hospital care is led by internal physicians without dedicated gastroenterology/hepatology training. Implementation of CDM models will also be helped by a more thorough understanding of the critical elements of successful models and protocolization of these elements. Examples of useful protocols used in our pilot trial were two self-management action plans for ascites and encephalopathy. Clearly the development of standardized training processes for inexperienced care coordinators, covering CDM and selfmanagement, will be required. Understanding and standardization of critical care processes will hopefully lead to robust CDM models, which will be less dependent on the individual skills of coordinators.

Dr. Volk also perceptively identifies the further problem of monitoring the effectiveness of CDM interventions in CLF performed outside of clinical trials. At their core, successful CDM models aim to improve patient outcomes by way of improvements in patient education, self-management, and access to quality care. We would suggest some of the following "process metrics" as indicators of improved patient engagement and successful implementation: medication adherence, improved self-management as assessed by validated tools, ${ }^{12}$ and attendance at planned outpatient care.

\section{Combined Authors Recommendations for CDM in Cirrhosis}

1. CDM models are likely to be effective in CLF, but the unique challenges of this condition require that high-quality randomized studies are performed.

2. Studies of CDM in cirrhosis must be appropriately powered for clinically relevant endpoints including mortality and cost effectiveness, be conducted in clinically relevant settings, and provide sufficient details about the nature of the intervention.

3. Translation of findings from such trials into routine clinical practice will require standardization of care processes and training, measurement of implementation success, and flexibility to adjust to different healthcare systems. 


\section{References}

1. Hoffman C, Rice D, Sung HY. Persons with chronic conditions. Their prevalence and costs. JAMA 1996;276:1473-1479.

2. Gines P, Quintero E, Arroyo V, Teres J, Bruguera M, Rimoa A, et al. Compensated cirrhosis: natural history and prognostic factors. HepatoLOGY 1987;7:122-128.

3. Volk ML, Tocco RS, Bazick J, Rakoski MO, Lok AS. Hospital readmissions among patients with decompensated cirrhosis. Am J Gastroenterol 2012;107:247-252.

4. Volk ML, Piette JD, Singal AS, Lok AS. Chronic disease management for patients with cirrhosis. Gastroenterology 2010;139:14-16.e1.

5. Wigg AJ, McCormick R, Wundke R, Woodman RJ. Efficacy of a chronic disease management model for patients with chronic liver failure. Clin Gastroenterol Hepatol 2013;11:850-858.e1-4.

6. Morando F, Maresio G, Piano S, Fasolato S, Cavallin M, Romano A, et al. How to improve care in outpatients with cirrhosis and ascites: a new model of care coordination by consultant hepatologists. J Hepatol 2013;59:257-264.
7. Weinberger M, Oddone EZ, Henderson WG. Does increased access to primary care reduce hospital readmissions? Veterans Affairs Cooperative Study Group on Primary Care and Hospital Readmission. N Engl J Med 1996;334:1441-1447.

8. Arora S, Thornton K, Murata G, Deming P, Kalisman S, Dion D, et al. Outcomes of treatment for hepatitis $\mathrm{C}$ virus infection by primary care providers. N Engl J Med 2011;364:2199-2207.

9. Dorn SD. Gastroenterology in a new era of accountability: part 3. Accountable care organizations. Clin Gastroenterol Hepatol 2011;9: 750-753.

10. Newhouse R, Bobay K, Dykes PC, Stevens KR, Titler M. Methodology issues in implementation science. Med Care 2013;51:S32-S40.

11. Volk ML, Fisher N, Fontana RJ. Patient knowledge about disease self-management in cirrhosis. Am J Gastroenterol 2013;108:302305.

12. Petkov J, Harvey P, Battersby M. The internal consistency and construct validity of the partners in health scale: validation of a patient rated chronic condition self-management measure. Qual Life Res 2010; 19:1079-1085. 